\title{
A atuação do enfermeiro na prevenção da Insuficiência Renal Crônica em pacientes com Hipertensão Arterial Sistemica
}

The nurse in the prevention of chronic renal failure in hypertensive patients

El papel de las enfermeras en la prevención de la insuficiencia renal crónica en pacientes con hipertensión arterial sistémica

Recebido: 09/11/2021 | Revisado: 14/11/2021 | Aceito: 15/11/2021 | Publicado: 25/11/2021

Ana Fátima Souza Melo de Andrade ORCID: https://orcid.org/0000-0002-7024-6175 Centro Universitário Estácio de Sergipe, Brasil E-mail: anafatimamelo@hotmail.com

Sthefany Rafaelly Freire Costa Lima ORCID: https://orcid.org/0000-0003-2442-3721 Centro Universitário Estácio de Sergipe, Brasil E-mail: sthefanyrfcl@gmail.com

Karine da Conceição Santos

ORCID: https://orcid.org/0000-0002-9273-5842 Centro Universitário Estácio de Sergipe, Brasil

E-mail: karineso106@gmail.com

Weber de Santana Teles

ORCID: https://orcid.org/0000-0003-1770-8278

Centro de Hemoterapia de Sergipe, Brasil

E-mail: arteecura@hotmail.com

Max Cruz da Silva

ORCID: https://orcid.org/0000-0002-6944-5986

Faculdade Pio Décimo, Brasil

E-mail: maxlfi@hotmail.com

Ruth Cristini Torres

ORCID: https://orcid.org/0000-0002-8664-192X Instituto de Hematologia e Hemoterapia de Sergipe, Brasil

E-mail: ruthcristini@gmail.com

Marcel Vinícius Cunha Azevedo

ORCID: https://orcid.org/0000-0002-5312-3333 Universidade Tiradentes, Brasil

E-mail: marcelvinicius49@gmail.com

Ângela Maria Melo Sá Barros

ORCID: https://orcid.org/0000-0003-4087-3247

Universidade Federal do Rio de Janeiro, Brasil

E-mail: angelsamelo@hotmail.com

Silvia Maria da Silva Sant'ana Rodrigues

ORCID: https://orcid.org/0000-0003-2421-8701

Centro Universitário Uninassau, Brasil

E-mail: profenf.silviasantana@gmail.com

Maria Hozana Santos Silva

ORCID: https://orcid.org/0000-0001-5742-5366

Faculdade Ages de Medicina, Brasil

E-mail: hosana_p@hotmail.com

Aline Barreto Hora

ORCID: https://orcid.org/0000-0002-3930-6475

Centro Universitário Estácio de Sergipe, Brasil

E-mail: aline.barretoh@ hotmail.com

Paulo Celso Curvelo Santos Junior

ORCID: https://orcid.org/0000-0001-5834-6782

Universidade Tiradentes, Brasil

E-mail: paulo.curvelo.jr@gmail.com

Taíssa Alice Soledade Calasans

ORCID: https://orcid.org/0000-0003-0460-4437

Universidade Tiradentes, Brasil

E-mail: taissa.asc@gmail.com

\section{Resumo}

O objetivo desse artigo é verificar, na literatura científica atual, as condutas que osenfermeiros prestam no atendimento aos usuários hipertensos para prevenção da insuficiência renal crônica. Trata-se de uma revisão integrativa executada no segundo semestre de 2020 nas bases da Biblioteca Virtual de Saúde. Foram incluídos artigos originais, em português, 
com texto completo disponível e publicados entre os anos de 2015 e 2020, totalizando uma amostra de 14 artigos. As principais ações do enfermeiro na assistência ao hipertenso na prevenção da Insuficiência Renal Crônica são: a identificação precoce, o controle dos fatores de risco, o debate acerca da situação do paciente entre todos os membros da equipe, a execução correta do processo de enfermagem e a verificação de fatores que possam atrapalhar a adesão do paciente. Conclui-se então que essas ações minimizam o risco de evolução da doença e se encaixam nas estratégias de promoção à saúde e prevenção de agravos.

Palavras-chave: Cuidados de enfermagem; Hipertensão; Insuficiência Renal Crônica; Prevenção de doenças.

\begin{abstract}
The objective of this article is to verify, in the current scientific literature, the behaviors that nurses provide in the care of hypertensive users for the prevention of chronic renal failure. This is an integrative review carried out in the second half of 2020 on the bases of the Virtual Health Library. Original articles were included, in Portuguese, with the full text available and published between the years 2015 and 2020, totaling a sample of 14 articles. The main actions of nurses in the care of hypertensive patients in the prevention of Chronic Kidney Failure are: early identification, control of risk factors, debate about the patient's situation among all team members, the correct execution of the nursing process and the verification of factors that may hinder patient compliance. It is therefore concluded that these actions minimize the risk of disease progression and fit into health promotion and disease prevention strategies.
\end{abstract}

Keywords: Nursing care; Hypertension; Chronic Kidney Failure; Prevention of diseases.

\title{
Resumen
}

El objetivo de este artículo es verificar, en la literatura científica actual, los comportamientos que brindan las enfermeras en la atención de los usuarios hipertensos para la prevención de la insuficiencia renal crónica. Se trata de una revisión integradora realizada en el segundo semestre de 2020 sobre las bases de la Biblioteca Virtual en Salud. Se incluyeron artículos originales, en portugués, con el texto completo disponible y publicados entre los años 2015 y 2020, totalizando una muestra de 14 artículos. . Las principales acciones del enfermero en la atención de pacientes hipertensos en la prevención de la Insuficiencia Renal Crónica son: identificación precoz, control de factores de riesgo, debate sobre la situación del paciente entre todos los integrantes del equipo, la correcta ejecución del proceso de enfermería y la verificación de factores que pueden obstaculizar el cumplimiento del paciente. Por tanto, se concluye que estas acciones minimizan el riesgo de progresión de la enfermedad y encajan en las estrategias de promoción de la salud y prevención de enfermedades.

Palabras clave: Atención de enfermería; Hipertensión; Insuficiencia renal crónica; Prevención de enfermedades.

\section{Introdução}

As doenças crônicas não transmissíveis (DCNT) são as principais causas de mortalidade no mundo. Dentre elas, a Hipertensão Arterial Sistêmica (HAS) é uma condição clinica multifatorial, que se define como o aumento da tensão dentrodos vasos sanguíneos (Jardim et al., 2017). Tal patologia tem como fatores predisponentes não modificáveis a idade, genética, gênero e etnia. Já em relação aos fatores modificáveis podem ser citados a obesidade, o consumo excessivo de sódio, etilismo, tabagismo, sedentarismo, estresse e fatores socioeconômicos (Paiva et al., 2016).

Em meio às consequências da hipertensão destacam-se a doença arterial coronária, a insuficiência cardíaca congestiva, a doença arterial periférica e a insuficiência renal. A Insuficiência Renal Crônica (IRC) ocorre, dentre outros motivos, devido à sobrecarga de volume e salina, que prejudicam o funcionamentocorreto dos rins a nível glomerular (Castro et al., 2019). Segundo os dados epidemiológicos, cerca de $35 \%$ da população brasileira é hipertensa, sendo que desse total, $4 \%$ desenvolve a Insuficiência Renal Crônica (Castro et al., 2018).

Desse modo, é importante destacar que os pacientes hipertensos, para nãodesenvolverem a IRC, necessitam de um atendimento de qualidade e um cuidado minucioso. Sabe-se que os indivíduos portadores de hipertensão e seus familiaressofrem muitas modificações na vida diária, sendo o conhecimento da equipemultiprofissional de grande importância na qualidade da assistência prestada (Marinho \& Thome, 2019).

A enfermagem deve então agir de maneira segura, prestando uma assistência de qualidade. Faz-se necessário entender as particularidades de cada paciente e elaborar condutas baseadas nas características próprias de cada indivíduo. Vale ressaltar que o enfermeiro deve possuir competência e habilidade para avaliar, prevenir e decidir quais condutas serão as mais adequadas 
para os pacientes com hipertensão, visando mitigar o risco do desenvolvimento da IRC (Rocha et al., 2015).

Considerando as comorbidades advindas a pacientes hipertensos e osagravos provocados pela IRC, o presente estudo se torna relevante à medida que procura elencar o trabalho do enfermeiro neste cenário a fim de prevenir e/ou minimizar os danos. A motivação para o desenvolvimento desta pesquisa se deu através da análise dos índices crescentes da IRC em pacientes hipertensos. Acredita-se que esta pesquisa possa contribuir para o aumento da produção científica dentro desta temática, servindo como base para ações pautadas em evdências concretas.

Com isso, o objetivo primordial desse artigo é verificar, na literatura científica atual, as condutas que os enfermeiros prestam no atendimento aos usuários hipertensos para prevenção da Insuficiência Renal Crônica.

\section{Metodologia}

O presente artigo trata-se de uma revisão integrativa da literatura, desenvolvida entre agosto e outubro de 2020. Esse tipo de estudo é uma ferramenta eficazpara garantir o desenvolvimento da assistência de enfermagem baseada em indícios científicos. Este recurso tem a capacidade de sintetizar os resultados encontrados em outros estudos sobre a mesma temática, direcionando o pesquisador a resultados com maior cientificidade. Sua confecção se dá em seis etapas: criação da questão norteadora, pesquisa da amostra na literatura, coleta de dados, análise dos dados, discussão dos resultados encontrados e montagem da revisão (Souza, Silva \& Carvalho, 2010).

A pesquisa foi baseada na seguinte questão norteadora: "Quais as ações do enfermeiro na assistência a pacientes hipertensos a fim de prevenir a Insuficiência Renal Crônica?". Optou-se por realizar a busca dos nos seguintes bancos de dados BVS- Biblioteca Virtual em Saúde, SCIELO-Scientific Eletronic Library Online, LILACS-Literatura Latino Americana e do Caribe em Ciências da Saúde, BDENF-Base de Dados em Enfermagem), utilizando para isso os descritores "Insuficiência Renal Crônica", "Cuidados de Enfermagem", "Hipertensão e Prevenção". Para fazer a correlação entre cada descritor, fez-se uso do operador booleano $A N D$ entre os termos.

Como critérios de inclusão, foram selecionados artigos originais, gratuitos, com texto completo disponível em português, publicados entre 2015 e 2020.Salienta-se que para o caso de artigos encontrados em mais de uma busca optou-se por selecionar o primeiro artigo, descartando a duplicidade.

O resultado das buscas contemplou 162 artigos que obedeciam aos critérios de inclusão. Depois de realizar uma leitura seletiva analisando os títulos eresumos, a fim de coletar aqueles cuja temática fosse pertinente ao objetivo dessapesquisa, foram definidos os estudos de real interesse. Dessa forma, obteve-se um total de 14 artigos válidos para responder a questão norteadora e alcançar o objetivo proposto.

Foi desenvolvido um formulário de coleta de dados, preenchido para cada artigo da amostra final do estudo com os seguintes itens: título, autores e ano de publicação, metodologia, considerações finais. Os artigos encontrados foram organizados conforme o ano de publicação de forma decrescente e os dados foram analisados, segundo os seus conteúdos, de forma descritiva.

Para análise dos dados, optou-se por utilizar o programa Microsoft Excel agrupando os resultados em gráficos. A presente pesquisa obedece aos padrões éticos fundamentados na lei $n^{\circ}$ 12853/2013 que regulamenta os direitos autorais.

\section{Resultados}

Após a seleção dos artigos para compor a amostra desse estudo, utilizou- se um instrumento de coleta de dados com o intuito de organizar sistematicamente as informações obtidas nos 14 artigos selecionados. Quanto aosperiódicos, foram identificados 10 diferentes, em destaque a Revista de Enfermagem UFPE, contribuindo com 04 estudos (28,57\%), e a Revista Enfermagem UERJ, com 02 estudos (14,28\%). As demais contribuíram cada uma com 01 estudo (7,14\%), respectivamente. Os resultados serão observados e descritos abaixo, conforme o Quadro 1. 
Quadro 1 - Instrumento para coleta das informações.

\begin{tabular}{|c|c|c|c|}
\hline Título doartigo & $\begin{array}{c}\text { Autores e Ano de } \\
\text { publicação }\end{array}$ & Metodologia & Considerações finais \\
\hline $\begin{array}{l}\text { Percepção da pessoa sobre sua condição } \\
\text { enquanto doente renal crônico em } \\
\text { hemodiálise }\end{array}$ & Santos, et al.,2020. & $\begin{array}{l}\text { Estudo qualitativo } \\
\text { exploratório }\end{array}$ & $\begin{array}{l}\text { Observou-se a importância da rede formal de saúde } \\
\text { para estes sujeitos, sendo a maioria portador de } \\
\text { comorbidades como hipertensão arterial sistêmica e } \\
\text { diabetes mellitus. }\end{array}$ \\
\hline $\begin{array}{l}\text { Política pública brasileira na prevenção } \\
\text { da doença renal crônica: desafos e } \\
\text { perspectivas }\end{array}$ & Silva, et al.,2020. & Estudo de caso & $\begin{array}{l}\text { As experiências brasileira e internacional } \\
\text { evidenciam que ações de prevenção da doença renal } \\
\text { crônica em grupos de risco ainda são incipientes, } \\
\text { especialmente em países de baixa renda. }\end{array}$ \\
\hline $\begin{array}{l}\text { Fatores associadosà doença renal crônica: } \\
\text { inquérito epidemiológico da Pesquisa } \\
\text { Nacional de Saúde }\end{array}$ & Aguiar, et al.,2020. & $\begin{array}{c}\text { Inquérito } \\
\text { Epidemiológico }\end{array}$ & $\begin{array}{l}\text { A prevalência de Doença Renal Crônica foi maior } \\
\text { em idade mais avançada, baixa escolaridade, possuir } \\
\text { plano de saúde, tabagismo, hipertensão, } \\
\text { hipercolesterolemia e avaliação regular ou ruim do } \\
\text { estado de saúde. }\end{array}$ \\
\hline $\begin{array}{l}\text { O enfermeiro e a questão da adesãodo } \\
\text { paciente ao tratamento da hipertensão } \\
\text { arterial } \\
\text { sistêmica }\end{array}$ & $\begin{array}{l}\text { Salles, et al., } \\
2019\end{array}$ & $\begin{array}{l}\text { Estudo Qualitativo } \\
\text { Descritivo }\end{array}$ & $\begin{array}{l}\text { O sistema utilizado na adesão ao tratamento é } \\
\text { similar entre as unidades de Estratégia em Saúde da } \\
\text { Família mantendo um padrão preconizado pela } \\
\text { literatura. }\end{array}$ \\
\hline $\begin{array}{l}\text { Efetividade da consulta de enfermagem } \\
\text { na adesão ao tratamento da hipertensão } \\
\text { arterialsistêmica }\end{array}$ & Mota \& Cortez, 2019. & $\begin{array}{l}\text { Ensaio Clínico Não } \\
\text { Controlado }\end{array}$ & $\begin{array}{l}\text { A assistência sistematizada de enfermagem pode } \\
\text { beneficiar pessoas diagnosticadas com hipertensão } \\
\text { arterial sistêmica naatenção primária em saúde. }\end{array}$ \\
\hline $\begin{array}{l}\text { Diagnósticos de enfermagem: disposição } \\
\text { para controle da saúde melhorado e } \\
\text { controle ineficaz dasaúde em hipertensos }\end{array}$ & $\begin{array}{l}\text { Moraes, et al., } \\
2019 .\end{array}$ & $\begin{array}{l}\text { Estudo Descritivo } \\
\text { Transversal }\end{array}$ & $\begin{array}{l}\text { Os diagnósticos de enfermagem disposição para } \\
\text { controle de saúdemelhorado e controle ineficaz da } \\
\text { saúde permitiram identificar a adesão dos } \\
\text { hipertensos ao tratamento, assim como a } \\
\text { necessidade de promover intervenção } \\
\text { multiprofissional que favoreça a assistência a estes } \\
\text { pacientes. }\end{array}$ \\
\hline $\begin{array}{l}\text { Significado das ações educativas na } \\
\text { consulta de enfermagem para clientes } \\
\text { renais crônicos e familiares }\end{array}$ & $\begin{array}{l}\text { Menezes, etal., } \\
2018 .\end{array}$ & $\begin{array}{l}\text { Estudo qualitativo } \\
\text { com base na } \\
\text { Fenomenologia } \\
\text { Social } \\
\end{array}$ & $\begin{array}{l}\text { O estudo permitiu compreender que, no cotidiano } \\
\text { desses indivíduos, elesadquirem habilidade e prática } \\
\text { para lidar com suas limitações. }\end{array}$ \\
\hline $\begin{array}{l}\text { Perfil sociodemográfico e clínico de } \\
\text { pacientesrenais crônicos em hemodiálise }\end{array}$ & $\begin{array}{l}\text { Piccin, et al., } \\
2018 .\end{array}$ & $\begin{array}{l}\text { Estudo Quantitativo } \\
\text { Descritivo }\end{array}$ & $\begin{array}{l}\text { Colabora-se, por meio do conhecimento do perfil } \\
\text { sociodemográfico e clínico dos pacientes que } \\
\text { realizam a hemodiálise, para o gerenciamento do } \\
\text { cuidado, que pode subsidiar estratégias para a } \\
\text { prevenção da doença e estimular a adesão ao } \\
\text { tratamento, além de contribuir para o avanço } \\
\text { científico em Nefrologia. }\end{array}$ \\
\hline $\begin{array}{l}\text { Perfil epidemiológico depacientes renais } \\
\text { crônicos em } \\
\text { tratamento }\end{array}$ & $\begin{array}{l}\text { Santos, et al., } \\
2018 .\end{array}$ & $\begin{array}{l}\text { Estudo Quantitativo } \\
\text { Descritivo }\end{array}$ & $\begin{array}{l}\text { Os dados identificados para a ocorrência de Doença } \\
\text { Renal Crônicapodem ser prevenidos, principalmente } \\
\text { no âmbito da atenção primária em saúde. }\end{array}$ \\
\hline $\begin{array}{l}\text { Acessibilidade ao tratamento da } \\
\text { hipertensão arterialna estratégia saúdeda } \\
\text { família }\end{array}$ & Rêgo, et al.,2018. & $\begin{array}{c}\text { Estudo } \\
\text { Transversal }\end{array}$ & $\begin{array}{l}\text { Os serviços ofertados às pessoas com hipertensão } \\
\text { arterial foram avaliados como regular, apresentando } \\
\text { principais barreiras referentes a aspectos } \\
\text { geográficos e organizacionais. }\end{array}$ \\
\hline $\begin{array}{l}\text { Sistematização da assistência } \text { de } \\
\text { enfermagem na atenção primária a } \\
\text { pessoas portadorasde hipertensão } \\
\text { arterial }\end{array}$ & $\begin{array}{l}\text { Pinto \& Rodrigues, } \\
2018 .\end{array}$ & $\begin{array}{l}\text { Estudo Descritivo } \\
\text { Transversal }\end{array}$ & $\begin{array}{l}\text { Conclui se que a sistematização da assistência ao } \\
\text { hipertenso em alto risco cardiovascular na Atenção } \\
\text { Primária representa uma metodologia para subsidiar } \\
\text { as açõesde promoção à saúde e prevenção } \\
\text { dos agravos. }\end{array}$ \\
\hline $\begin{array}{l}\text { Dificuldades de idosos na adesão ao } \\
\text { tratamento da hipertensão arterial }\end{array}$ & $\begin{array}{l}\text { Resende, et al., } \\
\quad 2018 .\end{array}$ & $\begin{array}{l}\text { Estudo Quantitativo } \\
\text { Descritivo }\end{array}$ & $\begin{array}{l}\text { Observou-se que os aspectos sociodemográficos, as } \\
\text { alterações do estado emocional, a falta de } \\
\text { acessibilidade e rede de apoio, a ausência de } \\
\text { sintomatologia da hipertensão arterial e a violência } \\
\text { urbana influenciaram a adesão àterapêutica por parte } \\
\text { dos idosos }\end{array}$ \\
\hline $\begin{array}{l}\text { Características sociodemográficas, clínicas } \\
\text { e subjetivas de clientes com doença renal } \\
\text { crônica atendidos na consulta de } \\
\text { enfermagem }\end{array}$ & $\begin{array}{l}\text { Menezes, et al., } \\
2017 .\end{array}$ & $\begin{array}{c}\text { Estudo } \\
\text { Quantiqualitativo }\end{array}$ & $\begin{array}{l}\text { O enfermeiro deve viabilizar e se responsabilizar } \\
\text { por intervenções que busquem entender a } \\
\text { complexidade do ser, respeitando cada característica } \\
\text { pessoal e se prontificando em associar cada } \\
\text { informação para uma melhor transmissão dos } \\
\text { aspectos do tratamento. }\end{array}$ \\
\hline $\begin{array}{l}\text { Detecção precocede doença renal crônica } \\
\text { em população de risco }\end{array}$ & $\begin{array}{l}\text { Dallacosta \& Mitrus, } \\
2017 .\end{array}$ & $\begin{array}{l}\text { Estudo Transversal } \\
\text { Epidemiológico }\end{array}$ & $\begin{array}{l}\text { Encontramos elevada prevalência dedoença renal, } \\
\text { especialmente no estágio dois, ratificando a } \\
\text { importância de orientação e adequado } \\
\text { acompanhamento dos hipertensos e diabéticos, } \\
\text { como formade estagnar a perda da função renal,e } \\
\text { trabalhar com foco na prevenção e promoção da } \\
\text { saúde. }\end{array}$ \\
\hline
\end{tabular}


A partir dessa organização dos dados, os resultados puderam ser compreendidos e caracterizados. Observa-se, por exemplo, que em relação à metodologia dos artigos, os estudos caracterizados como "Quantitativos Descritivos" e "Descritivo Transversal" obtiveram três artigos cada, sendo as duas principais metodologias encontradas. Pode-se observar a quantidade de todas as metodologias de pesquisa listadas pelos artigos da amostra demonstradas no Figura 1.

Figura 1 - Quantidade de metodologias.
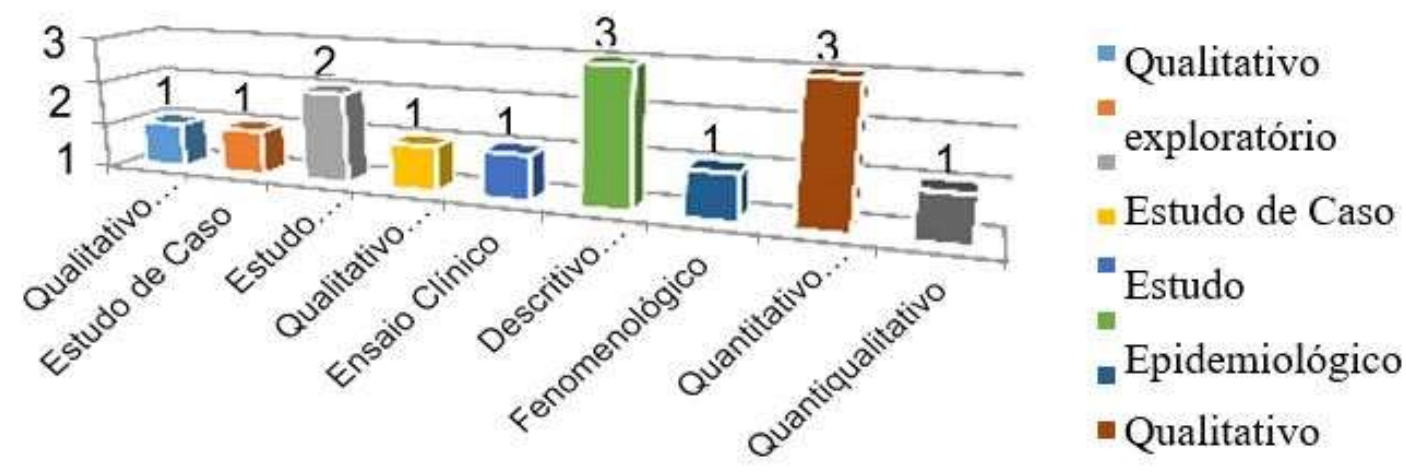

Fonte: Autores (2021).

Em relação ao ano de publicação de cada artigo, observou-se que não houve um padrão regular de distribuição. $\mathrm{O}$ ano de 2018 apresentou 06 artigos publicados e úteis para a presente pesquisa, revelando que nesse período os debates acerca das temáticas da Insuficiência Renal Crônica e da Hipertensão Arterial estavam em alta, assim demonstrado no Figura 2.

Figura 2 - Artigos de acordo com o ano de publicação.

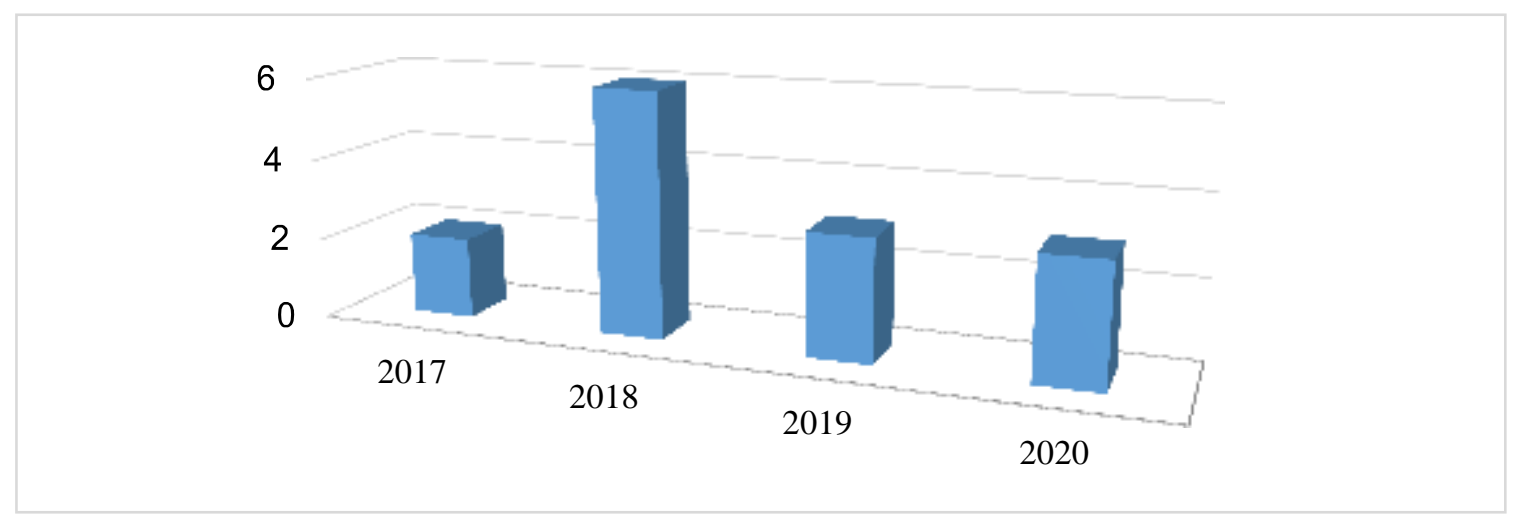

Fonte: Autores (2021).

Os artigos participantes da amostra deste estudo foram separados e caracterizados em duas categorias, visando facilitar a discussão das temáticas. A primeira, intitulada "Características dos pacientes com IRC" conta com 06 (43\%) dos artigos, enquanto a subsequente, "Ações do enfermeiro", conta com 08 (57\%) publicações, como descrito na Figura 3. 
Figura 3 - Caracterização dos artigos da amostra.

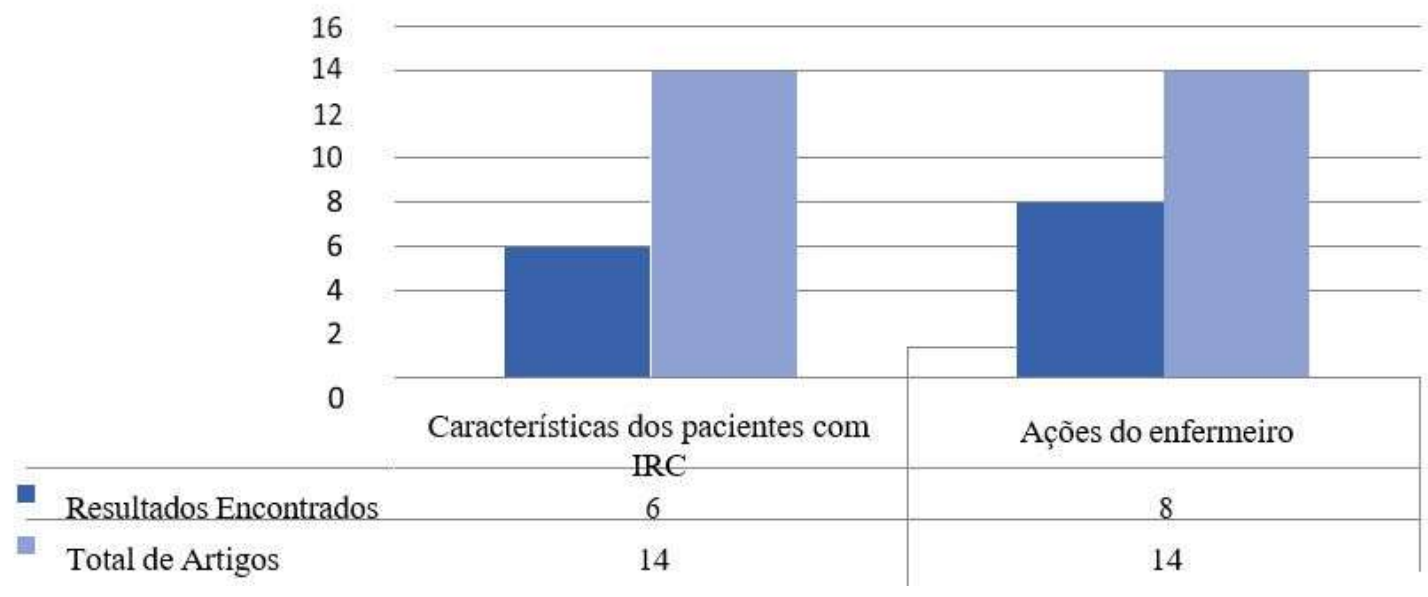

Fonte: Autores (2021).

\section{Discussão}

\subsection{Características dos pacientes com IRC}

Grande parte dos pacientes com IRC apresenta comorbidades como Hipertensão e Diabetes, além disso, ao perceberem o início dos sintomas, os pacientes procuraram imediatamente os serviços de saúde. Estes achados indicam que os profissionais de saúde devem estar atentos à progressão das comorbidades dos pacientes, a fim de prevenir complicações ocasionadas por doenças tratáveis na atenção básica (Santos, 2020).

Acerca do perfil desses pacientes, os estudos revelam que em sua maioria se tratam de homens, com idade próxima aos 50 anos e hipertensos (Piccin et al., 2018). Houve uma discordância no estudo de Menezes et al. (2017) que apontou o público feminino como principais portadoras de IRC. No entanto, percebe-se que o estudo em questão apresentou uma amostra inferior aos outros, o que leva a crer que os dados de gênero refletem um público local e não significativo para um perfil concreto.

A idade avançada e a presença de Hipertensão Arterial são fatores predominantes na população portadora de IRC. Ressalta-se ainda que o conhecimento da prevalência da IRC e dos fatores de risco e de proteção é essencial para prevenção da doença e para subsidiar as políticas públicas de saúde (Aguiar, 2020).

O envelhecimento da população aumenta a criação de grupos de risco para o surgimentos de patologias diversas. Concomitante a isso, as políticas públicas precisam avançar cada vez mais a fim de contemplar a saúde dessa população. Sendo o Sistema Único de Saúde a principal forma de tratamento para grande parte dos idosos, este deve ser estruturado de forma a permitir o rastreamento, identificação, tratamento e reabilitação do paciente. Além disso, a disseminação de informações é vital para aeducação não apenas dos pacientes, mas da sociedade como um todo (Silva, 2020).

O conhecimento é uma condição que promove a mudança de comportamento e muitas vezes retarda a progressão da doença. Enfatiza-se o direito de cidadania do paciente, ao ser atendido em suas necessidades de compartilhar, com os profissionais envolvidos, informações sobre seu estado de saúde para facilitar o autocuidado e sua participação no tratamento (Menezes, 2017).

\subsection{Ações do enfermeiro}

A identificação precoce da IRC é o primeiro passo que o enfermeiro deve dar no combate a esta patologia. Ressalta-se a importância da orientação e do adequado acompanhamento dos hipertensos e diabéticos, como forma de estagnar a perda da função renal. Dessa forma, é possível compreender que trabalhar com foco na prevenção e promoção da saúde minimiza os riscos àsaúde do paciente, diminuindo as chances de progressão das doenças de base (Dallacosta \& Mitrus, 2017). 
Identificando o paciente hipertenso, é possível realizar o controle dos fatores que levam a piora do quadro clínico. A abordagem terapêutica para o controle da pressão arterial na presença de DRC deve-se iniciar pelas medidas dietéticas e outras mudanças no estilo de vida, como a implementação de atividade física, a redução do consumo de bebidas alcoólicas e o abandono do tabagismo. Tais medidas são imprescindíveis para o controle da pressão arterial e também na redução do risco cardiovascular (Salles et al., 2019).

Colaborando com este pensamento, Rego et al. (2018) acrescentam que há necessidade de uma discussão mais aprofundada entre as equipes de Estratégia de Saúde da Família e gestores para a criação de novas estratégias de oferta de serviços para atender a demanda da população idosa, visando o atendimento de forma integral. Nesse ponto, o enfermeiro deve demonstrar um pensamento crítico- reflexivo acerca das necessidades de sua população adstrita, elaborando meios de facilitar o acesso dos usuários ao serviço de saúde.

Destarte, as ações executadas devem ser fundamentadas em princípios concretos e cientificamente comprovados. O trabalho de enfermagem sistematizado permite organizar e gerenciar a assistência ao paciente hipertenso na Atenção Primária à Saúde, cabendo ao enfermeiro implementar esta metodologia nas ações de promoção e prevenção desenvolvidas pela equipe de saúde, no intuito de amenizar os possíveis agravos a que estão expostos estes pacientes (Pinto \& Rodrigues, 2018).

Os fatores sociais (acessibilidade precária e violência urbana) e emocionais (medo do tratamento e falta de apoio da família) são os maioresimpedidores para a continuidade do tratamento. A fim de reduzir essa baixa adesão e prevenir o surgimento de complicações como a IRC, a instauração da Sistematização da Assistênciade Enfermagem é vital, tanto na captação desse público para as Unidades deSaúde quanto na criação de estratégias individuais eficazes (Resende \& Moraes, 2019).

\section{Considerações Finais}

O presente estudo abordou as condutas do enfermeiro diante dos pacientes portadores de Hipertensão Arterial Sistêmica (HAS), visando prevenir o desenvolvimento da IRC. Foi possível entender, através desta análise, que a maioria dos pacientes portadores da IRC são: homens, com idade avançada e que possuem a hipertensão e/ou diabetes como doença preexistente. Percebeu-se também que os pacientes que recebem instruções acerca do autocuidado apresentam melhores resultados no controle da patologia, o que significa uma qualidade de vida melhor.

Como principais ações do enfermeiro na assistência a pacientes com HAS, a fim de prevenir a IRC, destacam-se: a identificação precoce, o controle dos fatores de risco, o debate acerca da situação do paciente entre todos os membros da equipe, a execução correta do processo de enfermagem e a verificação de fatores que possam atrapalhar a adesão do paciente ao serviço de saúde. Essas ações minimizam o risco de evolução da doença e se encaixam nas estratégias de promoção à saúde e prevenção de agravos.

O enfermeiro desempenha um papel extremamente importante quanto as ações de educação em saúde, sensibilizando pascientes para o desenvolvimento do autocuidado a partir das ações educativas realizadas. Tais ações apresentam melhores resultados quando incorporadas também com a família do paciente, criando uma rede de apoio eficaz no tratamento de doenças crônicas.

Foi verificado na literatura a existência de um bom quantitativo de artigos falando sobre a HAS e sobre a IRC. No entanto, percebeu-se que poucas publicações abordavam os dois temas ao mesmo tempo, de maneira mais abrangente. Nesse caso, espera-se que essa pesquisa sirva como base para o desenvolvimento de novos estudos, garantindo a continuidade das pesquisascientíficas. 


\section{Referências}

Aguiar, L. K. (2020). Fatores associados à doença renal crônica: inquérito epidemiológico da Pesquisa Nacional de Saúde. Rev Bras Epidemiol. 23(1),1-15

Castro, M. C. M. (2019). Tratamento conservador de pacientes com doençarenal crônica que renuncia à dialise. Revista Braz. J. 41(1).

Castro, L. S. (2018). Perfil epidemiológico da hipertensão arterial sistêmica em uma população da zona urbana do Maranhão. Revista Eletrônica Acervo Saúde. $18(1)$.

Dallacosta, F. M., Dallacosta, H., \& Mitrus, L. (2017). Detecção precoce de doença renal crônica em população de risco. Cogitare Enferm. 22(2),1-6.

Jardim, L. M. S. S. V. (2017). Tratamento multiprofissional da hipertensão arterial sistêmica em pacientes muito idoso. Arq. Bras. Cardiol. 108(1).

Marinho, A. W. G. B. (2017). Prevalência de doença renal crônica em adultosno Brasil. Cad. Saúde Coletiva. 25(3).

Menezes, H. F. (2017). Características sociodemográficas, clínicas e subjetivasde clientes com doença renal crônica atendidos na consulta de enfermagem. Revista de enfermagem da UFPE. 11(5), 1858-1866.

Menezes, H. F (2018). Significado das ações educativas na consulta de enfermagem para clientes renais crônicos e familiares. Rev enferm UERJ, Rio deJaneiro. $26,1-8$.

Moraes, A. I. S. (2019). Diagnósticos de enfermagem: disposição para controle da saúde melhorado e controle ineficaz da saúde em hipertensos. Revista Cuidarte, 13(2), 111-115.

Mota, B. A. M., Lanza, F. M., \& Cortez, D. N. (2019). Efetividade da consulta de enfermagem na adesão ao tratamento da hipertensão arterial sistêmica. Rev. Salud Pública. 21(3), 1-9.

Paiva, M. M. (2016). Impacto da hipertensão arterial na qualidade de vida de idosos residentes na zona rural. Revista Enfermagem Atenção Saúde On- line. 5(1).

Piccin, C. (2018). Perfil sociodemográfico e clínico de pacientes renais crônicosem hemodiálise. Revista de enfermagem da UFPE. 12(12) 3212-3220.

Pinto, E. S. O., \& Rodrigues, W. M. (2018). Sistematização da Assistência de Enfermagem na Atenção Primária a pessoas portadoras de hipertensão arterial. Revista Nursing. 21(237), 2036-2040.

Rego, A. S. (2018). Acessibilidade ao tratamento da hipertensão arterial naestratégia saúde da família. Rev Gaúcha Enferm. 39, 1-9.

Resende, A. K. M. (2018). Dificuldades de idosos na adesão ao tratamento dahipertensão arterial. Revista de enfermagem da UFPE. 12(10), $2546-2554$.

Rocha, C. C. T. (2015). Hipertensão e diabéticos com insuficiência renal crônicano Brasil cadastrado no SIS/HIPERDIA. Revista Brasileira Hipertens, 22(1).

Salles, A. L. O. (2019). O enfermeiro e a questão da adesão do paciente ao tratamento da hipertensão arterial sistêmica. Rev enferm UERJ, Rio de Janeiro.27(2), $1-7$.

Santos, G. L. C. (2020). Percepção da pessoa sobre sua condição enquanto doenterenal crônico em hemodiálise. Rev Fun Care Online. 12 , 636-641.

Santos, K. K. (2018). Perfil epidemiológico de pacientes renais crônicos em tratamento. Revista de enfermagem da UFPE. 12(9), 2293-300.

Silva, P. A. B. (2020). Política pública brasileira na prevenção da doença renal crônica: desafios e perspectivas. Revista de Saúde Pública. 54(86),1-6.

Souza, M. T., Silva, M. D., \& Carvalho, R. (2010). Revisão integrativa: o que é e comofazer. Einstein. 8(1), $102-106$.

Thome, F. S. (2019) Inquérito brasileiro de diálise crônica 2017. Revista Braz. J. 41(1). 